\title{
The Research of Terminal Distribution Network Path Optimization
}

\author{
Xufeng Zhang1, Xinyu Zhang2 \\ ${ }^{1}$ International College, Beijing Wuzi University, Beijing, China \\ ${ }^{2}$ Graduate College, Beijing Wuzi University, Beijing, China \\ Email: xinyu715@126.com
}

Received 27 December 2013; revised 25 January 2014; accepted 3 February 2014

Copyright (C) 2014 by authors and Scientific Research Publishing Inc.

This work is licensed under the Creative Commons Attribution International License (CC BY).

http://creativecommons.org/licenses/by/4.0/

(c) (i)

\begin{abstract}
This paper presents an optimization problem about terminal distribution network path, defining the research problem through its distribution operation process, next to the terminal distribution route optimization. To begin with, dynamic optimization algorithm is built, the first from the target distribution node distribution vehicle, and goods to $\mathrm{N}$ customers are delivered, in the most appropriate distribution route to the minimum distribution distance and optimizing the terminal distribution path. Two parts to build and example are including algorithm.
\end{abstract}

\section{Keywords}

The Terminal Distribution; Dynamic Optimization Algorithm; Path Optimization

\section{Introduction}

In the process of logistic distribution network optimization, two stages are included: the first stage is the path optimization of distribution between nodes, namely the goods in the process of continuous distribution, the path between source node distribution to the target distribution optimization process; the second stage is the terminal distribution route optimization, namely by the target distribution node to the path optimization process between multiple clients. So this article mainly aims at terminal distribution route optimization problem. The terminal distribution route optimization is in the process of building dynamic optimization algorithm, from the target distribution node distribution vehicle, and delivering goods to $\mathrm{N}$ customers, in the most appropriate distribution route to the minimum distribution distance and optimizing the terminal distribution path. 


\section{Research Overview of the Logistics Distribution Network Path Optimization Problem}

\subsection{Distribution Network Path Optimization Research}

Path optimization problem in logistics distribution network with the traditional path optimization problem has certain difference, path optimization problems in network aiming at the condition of the traverse network point or edge can be divided into arc routing problem and point path problem. Among them, the arc routing problem consider is to service in the network edge, and some path problem is main consideration of neutral network service. A typical representative of the typical point path is traveling salesman problem and vehicle routing problem.

In view of the distribution network and its path optimization, Niu Yongliang [1] proposed a "three level" such as logistics distribution network structure, this structure with conform to the actual network structure of enterprise, on the principle of "quadtree" partition to the customer, using a two-phase heuristic algorithm to optimize the vehicle driving route. Wang Rumei [2], such as the freedom of the traditional annealing algorithm into directional annealing algorithm, and the examples show that this algorithm improves the efficiency. Jiang Zhongzhong [3], in the research of vehicle routing optimization problem, starting from the actual situation in the process of delivery, considering the uncertainty of vehicle travel time and customer service time, the logistics distribution network composed of distribution center and customer two kinds of nodes is not completely undirected graph representation, established a fuzzy programming model of logistics distribution vehicle routing optimization. Liu Qiusheng [4], such as vehicle loading optimization model is established, using the dynamic programming method to obtain the optimal solution.

Ko Hyun-Jeung and Geraldw. Evans (2007) [5] from the perspective of third-party logistics enterprises to build and optimize the mixed integer nonlinear programming model of reverse logistics, and puts forward the genetic algorithm based on heuristic algorithm to solve the model, Ma Zujun [6] from the Angle of the operating cost minimum, establishes the mixed integer linear programming model, in order to determine the number of facilities, location, and every reasonable logistics path through put.

H. A. Eliselt [7] mainly explores several categories such as: Chinese postman algorithm to the Chinese postal problems, have no to China post road, windy China postal problems, mixed China post road, heuristic China postal problems. G. Groves [8] study the profit maximization of China post road. W. L. Pharn [9] in combination with a variety of graph theory algorithm, at the same time considering the vehicle routing and scheduling management two aspects of the path optimization problem. Qin Wenqing [10], from the cooperation of supply chain level (horizontal), optimizes distribution network. The purpose is to improve the whole service level of the supply chain logistics network.

\subsection{Literature Review}

Most scholars in the research question will focus on the algorithm was improved, and focused on the goal of optimization design, but most of the studies did not fully combined with the actual distribution of the characteristics of the transportation network, most researchers think in between any two points can be directly to the model and algorithm on the basis of the design, so the logistics distribution route optimization problem with distribution of transportation network connectivity, remains to be further studied.

In most of these studies focused on the path of the logistics distribution network under normal conditions for optimization problems, due to the logistics distribution network not only include the traditional "positive” logistics distribution network, at the same time also includes return and old product recycling in the reuse of reverse logistics network, so in view of the combination of forward and reverse logistics distribution network in the path optimization problem remains to be further discussed.

\subsection{Research Purposes}

In view of the different nature and function of logistics distribution network nodes, the phases to solve the problem of logistics distribution network path optimization ideas and methods.

In logistics distribution network structure and based on the operation process of logistics distribution network, on the basis of logistics enterprises in the operating process of the path optimization of logistics distribution 
network can be divided into different stages. According to the characteristics of the second phase terminal distribution route optimization, constructing closed-loop dynamic optimization algorithm, and validate through the case.

\section{The Terminal Distribution Operation Process Ease}

1) Distribution location of dispatching personnel according to the customer, to customer order classification, divided into basic distribution area.

2) The nature of the goods, goods dispatching personnel according to user's need to classify the goods, will be the similar goods together, complete vehicle loading.

3) According to the required time to the customer order, determine the order of distribution, order of the identified here is tentative, because the further optimization needs according to the specific situation.

4) Operator according to the situation of the goods, the cost to arrange vehicles, according to the actual situation to choose outsourcing transportation or their own transport.

5) The operator according to the customer's position, the time of delivery requirements and local traffic condition, to determine the distribution line, as far as possible to meet customer request at the same time, the lowest transportation cost.

6) Determine after distribution line, according to the principle of minimum saving mileage, can determine the distribution order.

7) On the basis of certain distribution order, send before they are installed, after send first loading principle, loading, then transportation [11].

8) The customer sign for it, the owner customer and salesman, has its own team or other service providers to point the goods after the sign or seal on the delivery form, by a solicitor, has its own team or other service providers will bring the relevant papers and documents back. Distribution stage at the end of the operation flow chart shown in Figure 1.

From the point of view of the operation process of distribution between nodes, the key lies in the order in the shipping transit transfer process between the nodes, so in different constant goods distribution, the distribution of nodes by the source of goods distribution node to the target distribution by repeated movement-pause-sportspause. Through the analysis of enterprise logistics distribution system, can pause in the abstract into node, movement can be abstract as chain. Such goods distribution can be defined as the goods between distribution node in network of nodes and lines that connect the direction of the movement in the transfer process. From the operation process of the end of delivery, the key point is that the goods allocation process between customers, that is, from a process of distribution network at the grass-roots level to multiple clients. Because of the node of the two phase involves different, operation process is also different, so the path of the distribution network optimization problem was divided into different stages [12].

\section{Terminal Distribution Path Optimization Research}

\subsection{The Terminal Distribution Route Optimization Task Model}

The terminal distribution of the path optimization of logistics distribution network is goods from grassroots target distribution path optimization process between the node to multiple clients. The problem set from a primary distribution node from the system (1) is assumed to be node sends a vehicle to each customer within the system for material distribution, distribution again after the completion of the each customer returns and recycling materials back to the primary distribution node 1 . Research on material distribution vehicle shortest path and route. So it is a combination of forward logistics and reverse logistics of closed-loop path on closed-loop logistics network optimization process, as shown in Figure 1. Lines represent different nodes in the network transport of goods between the moving route, there may be multiple transport routes between any pair of nodes are linked together, these lines represent the different mode of transportation, transportation routes or different goods.

Mentioned in this paper, the closed-loop logistics refers to the full consideration in the process of traditional logistics distribution in reverse logistics, and put forward logistics and reverse logistics distribution system analysis and processing, make the logistics distribution system more reasonable, it is in the reverse logistics and forward logistics distribution is put forward on the basis of the combination of. As a result, the composition of closed-loop logistics network should include the following two parts: traditional "positive" logistics distribution 


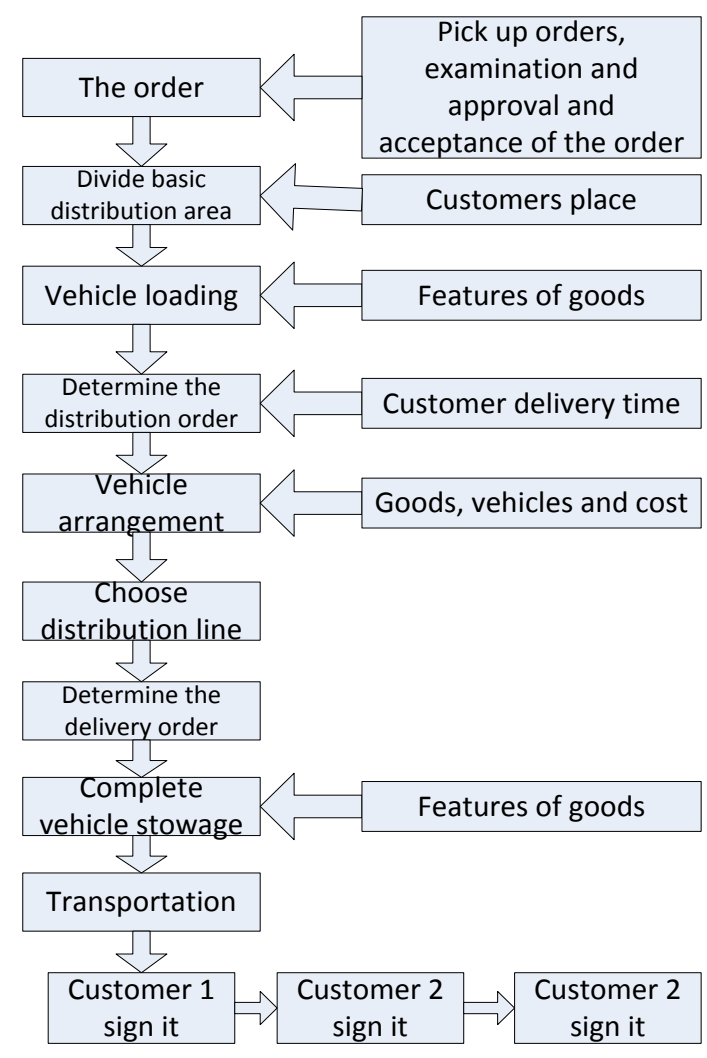

Figure 1. Operation procedure of distribution work.

network; Return and recycling of waste goods "reverse" logistics distribution network, two closed-loop characteristic of distribution network is mainly embodied in that they are not independent of each other but present a "from the source to the junction, again by the wired to source" characteristics. Therefore, under the traditional environment of "resources-production-consumption-abandoned" open loop process into a "resource-consumption-renewable resources" of the closed-loop feedback loop process. The generation of closed-loop logistics distribution is accorded with the requirement of sustainable development, and create new competitive advantage for the enterprise, it is not a simple forward logistics and reverse logistics and distribution network, but in the service of enterprise from the strategic level and operational level. Therefore, the environment and non-environment both form a closed loop of the emergence and development of logistics distribution [13]. As shown in Figure 2.

Because of the closed-loop logistics distribution system requires two-way logistics distribution, this increases the difficulty of the closed-loop logistics network planning, effective sharing facilities can effectively reduce logistics cost [14]. Due to the end customers are geographically dispersed, and have used materials have become much less the amount of the product, this to the forward logistics and reverse logistics distribution lines and the integration of transport capacity has brought the big difficulty, often provided by the integration of the discarded product cost is higher than output. Because of recycle and distribution have many differences, how to make effective and reasonable arrangement between product and waste product transport routes and traffic become a problem [15].

\subsection{The Terminal Distribution Route Optimization Algorithm Design}

\section{- Assumptions}

For the convenience of modeling and solving, in this paper, the real problem necessary to abstract and simplified. Specific assumptions as follows:

1) The definition of terminal distribution node: from a primary distribution node to multiple customer delivery; Primary distribution nodes and the location of the customer; the primary distribution node set the goods can 


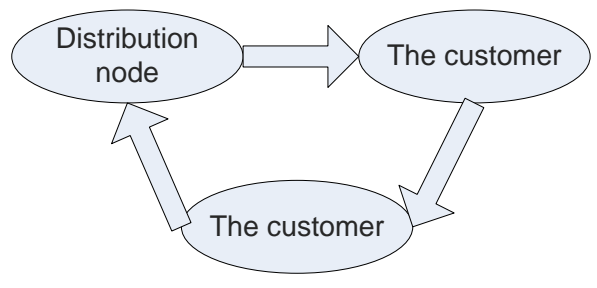

Figure 2. Closed distribution network.

reach its corresponding all customers.

2) The definition of vehicle loading conditions, the maximum load for each delivery vehicles that can be used and must be the same, does not allow overloading; Distribution, assuming that each pallet from primary distribution node, after completion of its corresponding customer service to return to the distribution center.

3) Definition to customer's requirement allows for different customer demand for the goods loaded in the same distribution inside the vehicle. Each customer's freight shall not exceed the maximum load distribution vehicle; Must satisfy each customer's delivery requirements, and can only be performed by a car, don't allow partial delivery; Customer demand for goods delivered without time window.

4) The definition of distribution area: consider only belongs to the scope of logistics distribution of a regional logistics.

5) The definition of the optimization goal: the shortest path of logistics distribution, distribution of the minimum cost and customer need the goods arrived in the shortest time belongs to the scope of time needed for the shortest path; In ignore the vehicle speed and vehicle operating cost difference under the premise of can think travel the shortest route is the most economical route.

6) The path definition: relevant information through the distance between two points to reflect the geographical position, without considering the limitation of other geographic facilities.

\section{- Basic Variable}

This section of the terminal distribution process involved need to traverse $\mathrm{N}$ customers, each customer needs service only once, the distance between two points is known, through the algorithm to find the end of the distribution process of the optimal path.

The algorithm of the variable parameter and their meanings:

$N_{i}=\{2,3, \cdots, i-1, i+1, \ldots, n\}$, said by distribution node 1 at the grass-roots level to the customer i may take a collection of intermediate nodes, $Q$ means by distribution node 1 at the grass-roots level to the customer i through a collection of intermediate nodes, $Q \in N_{i}$.

State variables said with $(i, Q)$, that distribution vehicle starting from the basic distribution node 1 , driving to the customer $i$, in the middle after the set of nodes for $Q$; $(\mathrm{i}, \theta)$ said delivery vehicle starting from the basic distribution node 1 ,drive directly to the customer $i$, in the middle without other clients, which said $\theta$ empty set.

The decision variables said $p_{k}\left(i_{k+1}, Q_{k}\right)=i_{k}$ decided to delivery vehicles are driven from customer $i_{k-1}$ to customer $i_{k}$.

The optimal value function said $f_{k}(i, Q)$ delivery from the primary distribution node 1 through $k$ intermediate nodes ( $Q$ said $K$ collection of intermediate nodes) to node $i$ of the shortest path distance.

\section{- Algorithm Design}

According to the basic assumptions of 3.2.1, in this article to solve the mathematical model of such as type (4-1), type (4-2).

$$
\begin{gathered}
f_{k}(i, Q)=\min _{j \in Q}\left\{f_{k-1}(j, Q /\{j\})+d_{j i}\right\}(k=1,2,3, \cdots, n-1 ; I=2,3, \cdots, n) \\
f_{0}(i, \theta)=d_{j i} \quad Q \in N_{i}
\end{gathered}
$$

Among them, the boundary conditions $f_{0}(i, \theta)$ said delivery vehicle starting from node 1 without intermediate node $(Q=\theta)$ directly to the distance $d_{j i}$ of the node $i$.

The optimal value function $f_{n-1}\left(1, N_{n, 1}\right), N_{n, 1}=\{2,3, \cdots, n\}$, is for delivery in the distribution network starting from node 1 to $n-1$ other intermediate node once and only once back in the most short distance node 1 . The 
best route of distribution $1=i_{0} \rightarrow i_{1} \rightarrow \cdots \rightarrow i_{k-1} \rightarrow i_{k} \rightarrow \cdots i_{n_{t}-1} \rightarrow i_{n_{t}}=1$ determined by the sequence of optimal decision $P_{1 n}^{*}=\left\{p_{0}\left(i_{1}, \theta\right), p_{1}\left(i_{2}, Q\right), p_{2}\left(i_{3}, Q_{2}\right), \cdots, p_{k}\left(i_{k+1}, Q_{k}\right), \cdots, p_{n-1}\left(i_{n}, Q_{n-1}\right)\right\}$ including the decision variables $p_{k}\left(i_{k+1}, Q_{k}\right)=i_{k}$ said distribution vehicle starting from the basic distribution node 1 , after $k$ middle customer once and only once ( $Q_{k}$ means $k$ middle customer collection) to the customer $i_{k+1}$ before tightening up the shortest route to a node $i_{k}$.

Through optimized step by step, finally can realize the terminal distribution optimization, such as type (4-3).

$$
\min Z=\sum_{i=1}^{n} f_{n-1}\left(1, N_{n, 1}\right)
$$

Among them $Z$ means the terminal distribution in the process of distribution vehicle distance sum.

\subsection{The Terminal Distribution Route Optimization Example}

\section{- Terminal distribution route optimization example background}

A closed-loop logistics network as shown in Figure 3, one is the logistics distribution network in A subordinate five commodity supermarket distribution node at the grassroots level on A daily basis at the grass-roots level distribution node sends A delivery goods distribution to each supermarket, and then will return after unloading and recycled goods loading, in order to the next destination, finally will return and recycled back to the primary distribution node. Distribution at the grass-roots level in Table 1 nodes and the distance between the five supermarket (unit: kilometers), delivery requirements from the primary distribution node after each supermarket once and only once, finally back to the primary distribution nodes, the optimal route for delivery of the car is driven and the shortest distance.

\section{- Terminal distribution route optimization example}

We put the grassroots distribution node supermarket $1, \ldots$, supermarket 5 in accordance with the number of node $1,2, \ldots, 6$, and by the question set conditions $d_{i j} \neq d_{j i}, I \neq j ; I, j=1,2, \ldots$, the distribution system of the road map is a directed multiple connected graph. According to the above discussion, we adopt the method of paramagnetic push logistics distribution vehicle driving the optimal route and shortest distance: $K=0$, the boundary conditions such as type (4-4). (The results are shown in Table 2.

$$
f_{0}(2, \theta)=d_{12}=15
$$

$p_{0}(2, \theta)=1, \mathrm{~K}=1$, such as type (4-5), (The results are shown in Tables 3-7).

$$
f_{1}(2,\{3\})=\min \left\{f_{0}(3, \theta)+d_{32}\right\}=26+16=42
$$

$p_{1}(6,\{2\})=2, K=2$, such as type (4-6), (The results are shown in Tables 8-12)

$$
f_{2}(2,\{3,4\})=\min \left\{f_{1}(3,\{4\})+d_{32}, f_{1}(4,\{3\})+d_{42}\right\}=\min \{43+16=59,38+36=74\}=59
$$

$p_{2}(5,\{2,6\})=6, K=3$ such as type (4-7), (The results are shown in Tables 13-17).

$$
\begin{aligned}
f_{3}(2,\{3,4,5\}) & =\min \left\{f_{2}(3,\{4,5\})+d_{32}, f_{2}(4,\{3,5\})+d_{42}, f_{2}(5,\{3,4\})+d_{52}\right\} \\
& =\min \{66+16=82,58+36=94,54+36=90\}=82
\end{aligned}
$$

$p_{3}(4,\{2,5,6\})=5, \mathrm{~K}=4$, such as type (4-8), (The results are shown in Tables 4-18)

$$
\begin{aligned}
f_{4}(2,\{3,4,5,6\}) & =\min \left\{f_{3}(3,\{4,5,6\})+d_{32}, f_{3}(4,\{3,5,6\})+d_{42}, f_{3}(5,\{3,4,6\})+d_{52}, f_{3}(6,\{3,4,5\})+d_{62}\right\} \\
& =\min \{94+16=110,79+36=115,80+36=116,82+28=110\}=11
\end{aligned}
$$

$p_{4}(3,\{2,4,5,6\})=4, \mathrm{~K}=5$, such as type (4-9) and (4-10),

$$
f_{5}(1,\{2,3,4,5,6\})=\min \left\{f_{4}(2,\{3,4,5,6\})+d_{21}, f_{4}(3,\{2,4,5,6\})+d_{31}, f_{4}(4,\{2,3,5,6\})+d_{41}\right\},
$$




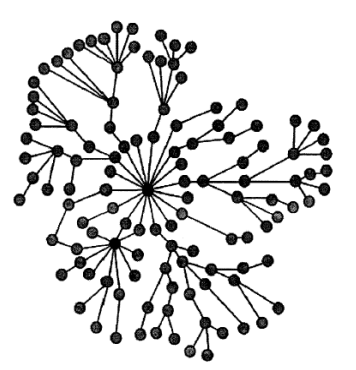

Figure 3. One of distribution network.

Table 1. Distribution distance between the nodes.

\begin{tabular}{|c|c|c|c|c|c|c|c|}
\hline \multirow[b]{2}{*}{ Node } & Node & Node 1 & Node 2 & Node 3 & Node 4 & Node 5 & Node 6 \\
\hline & & Disk-center & Market 1 & Market 2 & Market 3 & Market 4 & Market 5 \\
\hline Node 1 & Disk-center & 0 & 16 & 26 & 33 & 40 & 58 \\
\hline Node 2 & Market 1 & 18 & 0 & 25 & 36 & 31 & 26 \\
\hline Node 3 & Market 2 & 28 & 16 & 0 & 12 & 16 & 19 \\
\hline Node 4 & Market 3 & 38 & 36 & 10 & 0 & 16 & 25 \\
\hline Node 5 & Market 4 & 55 & 36 & 18 & 16 & 0 & 28 \\
\hline Node 6 & Market 5 & 66 & 28 & 20 & 29 & 18 & 0 \\
\hline
\end{tabular}

Table 2. Rout optimization table $(\mathrm{K}=0)$.

\begin{tabular}{ccccc}
\hline$f_{0}(2, \theta)$ & $f_{0}(3, \theta)$ & $f_{0}(4, \theta)$ & $f_{0}(5, \theta)$ & $f_{0}(6, \theta)$ \\
\hline$d_{12}$ & $d_{13}$ & $d_{14}$ & $d_{15}$ & $d_{16}$ \\
16 & 26 & 33 & 40 & 58 \\
\hline
\end{tabular}

Table 3. Rout optimization table $(\mathrm{K}=1, \mathrm{i}=2)$.

\begin{tabular}{cccc}
\hline$f_{1}(2,\{3\})$ & $f_{1}(2,\{4\})$ & $f_{1}(2,\{5\})$ & $f_{1}(2,\{6\})$ \\
\hline$f_{0}(3, \theta)+d_{32}$ & $f_{0}(4, \theta)+d_{42}$ & $f_{0}(5, \theta)+d_{52}$ & $f_{0}(6, \theta)+d_{62}$ \\
42 & 69 & 76 & 86 \\
\hline
\end{tabular}

Table 4. Rout optimization table $(\mathrm{K}=1, \mathrm{i}=3)$.

\begin{tabular}{cccc}
\hline$f_{1}(3,\{2\})$ & $f_{1}(3,\{4\})$ & $f_{1}(3,\{5\})$ & $f_{1}(3,\{6\})$ \\
\hline$f_{0}(2, \theta)+d_{23}$ & $f_{0}(4, \theta)+d_{43}$ & $f_{0}(5, \theta)+d_{53}$ & $f_{0}(6, \theta)+d_{63}$ \\
41 & 43 & 58 & 78
\end{tabular}

Table 5. Rout optimization table $(K=1, i=4)$.

\begin{tabular}{cccc}
\hline$f_{1}(4,\{2\})$ & $f_{1}(4,\{3\})$ & $f_{1}(4,\{5\})$ & $f_{1}(4,\{6\})$ \\
\hline$f_{0}(2, \theta)+d_{24}$ & $f_{0}(3, \theta)+d_{34}$ & $f_{0}(5, \theta)+d_{54}$ & $f_{0}(6, \theta)+d_{64}$ \\
52 & 38 & 56 & 87 \\
\hline
\end{tabular}

Table 6. Rout optimization table $(\mathrm{K}=1, \mathrm{i}=5)$.

\begin{tabular}{cccc}
\hline$f_{1}(5,\{2\})$ & $f_{1}(5,\{3\})$ & $f_{1}(5,\{4\})$ & $f_{1}(5,\{6\})$ \\
\hline$f_{0}(2, \theta)+d_{25}$ & $f_{0}(3, \theta)+d_{35}$ & $f_{0}(4, \theta)+d_{45}$ & $f_{0}(6, \theta)+d_{65}$ \\
47 & 42 & 56 & 76 \\
\hline
\end{tabular}


Table 7. Rout optimization table $(\mathrm{K}=1, \mathrm{i}=6)$.

\begin{tabular}{cccc}
\hline$f_{1}(6,\{2\})$ & $f_{1}(6,\{3\})$ & $f_{1}(6,\{4\})$ & $f_{1}(6,\{5\})$ \\
\hline$f_{0}(2, \theta)+d_{26}$ & $f_{0}(3, \theta)+d_{36}$ & $f_{0}(4, \theta)+d_{46}$ & $f_{0}(5, \theta)+d_{56}$ \\
42 & 45 & 58 & 68
\end{tabular}

Table 8. Rout optimization table $(\mathrm{K}=2, \mathrm{i}=2)$.

\begin{tabular}{rrrrrr}
\hline$f_{2}(2,\{3,4\})$ & $f_{2}(2,\{3,5\})$ & $f_{2}(2,\{3,6\})$ & $f_{2}(2,\{4,5\})$ & $f_{2}(2,\{4,6\})$ & $f_{2}(2,\{5,6\})$ \\
\hline 59 & 68 & 73 & 92 & 86 & 96 \\
\hline
\end{tabular}

Table 9. Rout optimization table $(\mathrm{K}=2, \mathrm{i}=3)$.

\begin{tabular}{cccccc}
\hline$f_{2}(3,\{2,4\})$ & $f_{2}(3,\{2,5\})$ & $f_{2}(3,\{2,6\})$ & $f_{2}(3,\{4,5\})$ & $f_{2}(3,\{4,6\})$ & $f_{2}(3,\{5,6\})$ \\
\hline 62 & 65 & 62 & 66 & 78 & 8 \\
\hline
\end{tabular}

Table 10. Rout optimization table $(K=2, \mathrm{i}=3)$.

\begin{tabular}{cccccc}
\hline$f_{2}(4,\{2,3\})$ & $f_{2}(4,\{2,5\})$ & $f_{2}(4,\{2,6\})$ & $f_{2}(4,\{3,5\})$ & $f_{2}(4,\{3,6\})$ & $f_{2}(4,\{5,6\})$ \\
\hline 53 & 61 & 71 & 58 & 74 & 92 \\
\hline
\end{tabular}

Table 11. Rout optimization table $(K=2, \mathrm{i}=4)$.

$\begin{array}{cccccc}f_{2}(5,\{2,3\}) & f_{2}(5,\{2,4\}) & f_{2}(5,\{2,6\}) & f_{2}(5,\{3,4\}) & f_{2}(5,\{3,6\}) & f_{2}(5,\{4,6\}) \\ 57 & 68 & 60 & 54 & 63 & 76\end{array}$

Table 12. Rout optimization table $(K=2, \mathrm{i}=5)$.

\begin{tabular}{cccccc}
\hline$f_{2}(6,\{2,3\})$ & $f_{2}(6,\{2,4\})$ & $f_{2}(6,\{2,5\})$ & $f_{2}(6,\{3,4\})$ & $f_{2}(6,\{3,5\})$ & $f_{2}(6,\{4,5\})$ \\
\hline 60 & 77 & 75 & 62 & 70 & 81 \\
\hline
\end{tabular}

Table 13. Rout optimization table $(\mathrm{K}=2, \mathrm{i}=2)$.

\begin{tabular}{cccc}
\hline$f_{3}(2,\{3,4,5\})$ & $f_{3}(2,\{3,4,6\})$ & $f_{3}(2,\{3,5,6\})$ & $f_{3}(2,\{4,5,6\})$ \\
\hline 82 & 90 & 98 & 109 \\
\hline
\end{tabular}

Table 14. Rout optimization table $(\mathrm{K}=3, \mathrm{i}=3)$.

$\begin{array}{cccc}f_{3}(3,\{2,4,5\}) & f_{3}(3,\{2,4,6\}) & f_{3}(3,\{2,5,6\}) & f_{3}(3,\{4,5,6\}) \\ 71 & 81 & 78 & 94\end{array}$

Table 15. Rout optimization table $(K=3, \mathrm{i}=4)$.

\begin{tabular}{cccc}
\hline$f_{3}(4,\{2,3,5\})$ & $f_{3}(4,\{2,3,6\})$ & $f_{3}(4,\{2,5,6\})$ & $f_{3}(4,\{3,5,6\})$ \\
\hline 73 & 74 & 76 & 79 \\
\hline
\end{tabular}

Table 16. Rout optimization table $(K=3, \mathrm{i}=4)$.

$\begin{array}{cccc}f_{3}(5,\{2,3,4\}) & f_{3}(5,\{2,3,6\}) & f_{3}(5,\{2,4,6\}) & f_{3}(5,\{3,4,6\}) \\ 69 & 78 & 87 & 80\end{array}$

Table 17. Rout optimization table $(K=3, \mathrm{i}=6)$.

$\begin{array}{cccc}f_{3}(6,\{2,3,4\}) & f_{3}(6,\{2,3,5\}) & f_{3}(6,\{2,4,5\}) & f_{3}(6,\{3,4,5\}) \\ 78 & 84 & 86 & 82\end{array}$


Table 18. Rout optimization table $(\mathrm{K}=4)$.

\begin{tabular}{cccc}
\hline$f_{4}(3,\{2,4,5,6\})$ & $f_{4}(4,\{2,4,5,6\})$ & $f_{4}(5,\{2,4,5,6\})$ & $f_{4}(6,\{2,4,5,6\})$ \\
\hline 86 & 90 & 90 & 90 \\
\hline
\end{tabular}

$$
\begin{gathered}
f_{4}(5,\{2,3,4,6\})+d_{51}, f_{4}(6,\{2,3,4,5\})+d_{61} \\
=\min \{110+18=128,86+28=114,90+38=128,90+55=145,90+66=156\}=114 \\
p_{5}(1,\{2,3,4,5,6\})=3
\end{gathered}
$$

Known from results on the analysis of the calculation, through the process of terminal distribution, the optimal route distribution vehicle: (1) - (2) - (6) - (5) - (4) - (3) - (1), the minimum distance is $114 \mathrm{~km}$.

\section{Summarizes}

Based on the logistics distribution network structure and the logistics distribution network based on the operation process, according to the distribution network at the grass-roots level to the customer the characteristics of the stage of transport mode, terminal logistics distribution network is proposed, and then combined with the characteristics of this stage transport path optimization of logistics distribution network.

In terminal distribution optimization of path optimization process, to build a logistics distribution, including traditional "positive" at the same time, also including the return and recycling of waste goods reverse logistics of closed-loop dynamic optimization algorithm, using the closed-loop dynamic optimization algorithm for the enterprise logistics distribution route optimization to grass-roots distribution nodes are studied, to determine the best path distribution. Validated, the calculated result is consistent with actuality.

\section{References}

[1] Niu, Y.L. and Wang, J.M. (2006) Logistics Distribution Vehicle Route Algorithm. Journal of Transportation Engineering, 6, 83-87.

[2] Wang, R.M., Wang, S.M. and Wang, Z.J. (2007) Directional Simulated Annealing Algorithm Is a Kind of Vehicle Routing Problem. Manufacturing Technology Research, 1, 16-19.

[3] Jiang, Z.Z. and Wang, D.W. (2006) And Fuzzy Planning Model of Logistics Distribution Vehicle Routing Optimization Algorithm. Journal of System Simulation, 17, 3301-3304.

[4] Liu, Q.S., Wen, X.J. and Pan, X.X. (2009) Home Appliances to the Countryside in the Logistics Distribution System Optimization of Vehicle Loading Study. Commercial Modernization, 575, 119-120.

[5] Hyun, J.K. and Gerald, W.E. (2007) A Genetic Algorithm-Based Heuristic for the Dynamic Integrated forward/ Reverse Logistics Network for 3PLs. Computers \& Operations Research, 34, 346-366. http://dx.doi.org/10.1016/j.cor.2005.03.004

[6] Ma, Z.J. (2005) Generation of Clever. Products Recycling Reverse Logistics Network Optimization Design Model. Journal of Management Engineering, 12, 114-117.

[7] Eiselt, H.A., Michel, G. and Gilbert, L. (1995) Arc Routing Problems, Part I: The Chinese Postman Problem. Operations Research, 43, 231-242. http://dx.doi.org/10.1287/opre.43.2.231

[8] Groves, G., le Roux, J. and van Vuuren, J.H. (2004) Network Service Scheduling and Routing. International Transactions in Operational, 11, 613-643.

[9] Pharn, W.L. and Chiu, W.C. (2005) Approximate Solutions for the Maximum Benefit Chinese Postman Problem. International Journal of Systems Science, 36, 815-822. http://dx.doi.org/10.1080/00207720500282292

[10] Qin, W.Q. (2006) Horizontal Cooperation Supply Chain and Distribution Network Research. Guangxi University.

[11] Mou, L.M. and Dai, X.D. (2013) Effective in Solving Selective Single Commodity Distribution Collection of Ant Colony Algorithm. Journal of Computer Applications and Software, 30, 93-96.

[12] Zhou, Y.C. and Sun, X.C. (2012) Logistics Distribution Path Optimization Research Based on Improved Genetic Algorithm. Computer Engineering and Science, 10, 118-122.

[13] Long, H. and Wu, Y. (2012) Time-Varying under the Condition of City Logistics Distribution Vehicle Routing Optimization. Journal of Automobile Industry of Hubei Province College, 2, 42-45. 
[14] Wang, N.L. (2012) The Automobile Assembly Line Material Distribution with Time Windows Path Planning. Journal of Industrial Engineering, 15, 94-99.

[15] Xu, L.R. (2013) Based on LINGO of City Logistics Distribution Path Optimization. Journal of Electronic Design Engineering, 276, 52-54. 